\title{
Reliability of joint angle during sit-to-stand movements in persons with stroke using portable gait analysis system based wearable sensors
}

\author{
Jung-Ae $A n^{a}$, Byoung-Hee Lee ${ }^{b}$ \\ ${ }^{a}$ Department of Physical Therapy, Seoul Now Hospital, Seongnam, Republic of Korea \\ ${ }^{b}$ Department of Physical Therapy, College of Health Science and Social Welfare, Sahmyook University, Seoul, Republic of Korea
}

Objective: The purpose of this study was to investigate the test-retest reliability and concurrent validity of the joint angle of the lower extremities during sit-to-stand movements with wearable sensors based on a portable gait analysis system (PGAS), and the results were compared with a analysis system (MAS) to predict the clinical potential of it.

Design: Cross-sectional study.

Methods: Sixteen persons with stroke ( 9 males, 7 females) participated in this study. All subjects had the MAS and designed PGS applied simultaneously and eight sensor units of designed PGAS were placed in a position to avoid overlap with the reflexive markers from MAS. The initial position of the subjects was $90^{\circ}$ of hip, knee, and ankle joint flexion while sitting on a chair that was armless and backless. The height of the chair was adjusted to each individual. After each trial, the test administrator checked the quality of data from both systems that measured sit-to-stand for test-retest reliability and concurrent validity.

Results: As a result, wearable sensor based designed PGAS and MAS demonstrated reasonable test-retest reliability for the assessment of joint angle in the lower extremities during sit-to-stand performance. The intra-class correlation coefficients (ICCs) for wearable sensor based designed PGAS showed an acceptable test-retest reliability, with ICCs ranging from 0.759 to 0.959 . In contrast, the MAS showed good to excellent test-retest reliability, with ICCS ranging from 0.811 to 0.950 . In concurrent validity, a significant positive relationship was observed between PGAS and MAS for variation of joint angle during sit-to-stand movements $(p<0.01)$. A moderate to high relationship was found in the affected hip ( $\mathrm{r}=0.665)$, unaffected hip ( $\mathrm{r}=0.767)$, affected knee $(\mathrm{r}=0.876)$, unaffected knee $(\mathrm{r}=0.886)$, affected ankle $(\mathrm{r}=0.943)$ and unaffected ankle $(\mathrm{r}=0.823)$ respectively.

Conclusions: The results of this study indicated that wearable sensor based designed PGAS showed acceptable test-retest reliability and concurrent validity in persons with stroke for sit-to-stand movements and wearable sensors based on developed PGAS may be a useful tool for clinical assessment of functional movement.

Key Words: Analysis, Movement, Position, Stroke

\section{Introduction}

Sit-to-stand (STS) performance is important ability for daily activities such as transfer, ambulation, toileting, and stair climbing in healthy individuals particularly for persons with stroke [1]. In addition, STS ability is intimately related with gait and provides further functional self-support [2,3].
The STS test has been used for stroke survivors to predict their balance ability and fall risk and training of STS is routinely performed in the rehabilitation unit. Several researchers investigated that the effects of STS training on balance in persons affected with stroke and have shown improvements $[4,5]$ and plenty of evidence of interventions for improving STS ability has been suggested, however the quality of mea-

Received: 29 August, 2019 Revised: 17 September, 2019 Accepted: 18 September, 2019

Corresponding author: Byoung Hee Lee (ORCID https://orcid.org/0000-0001-9766-6068)

Department of Physical Therapy, College of Health Science and Social Welfare, Sahmyook University, 815 Hwarang-ro, Nowon-gu, Seoul 01795, Republic of Korea

Tel: 82-2-3399-1634 Fax: 82-2-3399-1639 E-mail: 3679@syu.ac.kr

(c) This is an Open-Access article distributed under the terms of the Creative Commons Attribution Non-Commercial License (http://creativecommons.org/licenses/ by-nc/4.0) which permits unrestricted non-commercial use, distribution, and reproduction in any medium, provided the original work is properly cited.

Copyright (๑ 2019 Korean Academy of Physical Therapy Rehabilitation Science 
surements are unclear [6]. With altered knee joint angles, varying amounts and levels of plantar pressure affects STS pattern on the affected side [7].

To assess STS performance, a variety of assessment tools have been used but researchers still prefer three-dimensiona (3D) motion capture systems based on image due to their accuracy aside from their uncomfortable features such as specialized gait analysis laboratory environment, painful cost, and the post processing period [8]. In recent years, the limitations of the motion capture systems lead to diverse approaches to developing wearable sensors and created a great deal of improvement. Furthermore, wearable sensors, which are wearable, extensible, and affordable, may more conveniently provide useful information in the field of stroke rehabilitation [9].

The gait analysis system based on wearable sensors may estimate static and dynamic kinematic data. In this study, the focus was placed on static kinematic data measurement, especially during STS movements, and variation of joint angles in the lower body segment of stroke survivors.

The purpose of this study was to investigate the reliability joint angles in the lower extremity in persons with stroke during STS movements with the wearable sensor-based portable gait analysis system (PGAS), and the results were compared with motion capture system in order to be able to predict its potential to serve as a valuable clinical measurement.

\section{Methods}

\section{Participants}

Twenty persons with stroke were initially recruited from a local rehabilitation hospital, however, two potential participants did not meet the experimental criteria and two participants withdrew during the experimental trials. For that rea- son, only sixteen subjects were participated in this study. The inclusion criteria were stroke survivors who were at least six months post stroke and those able to understand the given instruction during the experimental procedure [10]. Subjects who had a Modified Mini Mental State Examination-Korea score of under 24, comprehensive aphasia, neglect, medical instability such as uncontrolled hypertension, and arrhythmia were excluded [11].

In addition, all procedures involved in the experimental protocol were explained to each participant and were approved by the Institutional Review Board of Sahmyook University (SYUIRB2014-092). All subjects provided written informed consent prior to enrollment in this study.

\section{Procedures}

The motion analysis system (MAS) and wearable sensor-based PGAS were simultaneously applied on the participant and eight sensor units of designed PGAS were located without overlapping the reflective markers of the MAS. The initial position of subject was $90^{\circ}$ angle of hip and knee flexion while sitting on a chair and $90^{\circ}$ angle of ankle dorsiflexion with foot placed on the ground. The chair which was an armless, backless chair that was firmly fixed to the floor, and the height was adjustable for each individual [12]. Eight sensor units, which were consisted of a wireless tri-axial accelerometer and gyroscope sensors, were attached on the lower extremities with straps for measurement of variation of joint angles during the sit to stand performance [13]. Markers, which enabled the Visual 3D (C-Motion, Rockville, MD, USA, 2012) to reconstruct the digital skeleton of the subject and the sensor units from the designed PGAS were attached by the identical physical therapist. Eight sensor units were placed in a position to avoid overlap with reflective markers from the motion capture system. All subjects performed three trials of sit to stand, which con-
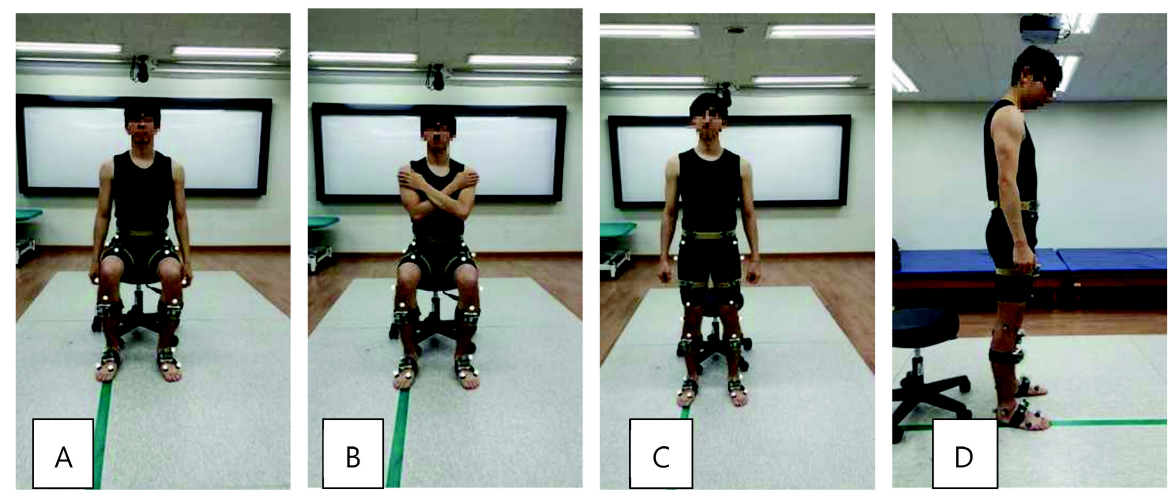

Figure 1. Experimental procedure. (A) Sitting. (B) Crossed arm. (C) Standing up. (D) Side-view. 
sisted of one practice trial and a repetition of two more trials (Figure 1). After each trial, the test administrator checked the quality of measured data during sit to stand for test-retest reliability.

Four skilled physical therapists that had received training for the experimental protocol had supervised the patient during the experimental procedure and two test administrators had checked the data from each system. Before the experiment, leg lengths (from great trochanter to lateral epicondyle, from lateral epicondyle to heel though the lateral malleolus) were measured. Eight sensor units were placed on the anterior superior iliac spine (ASIS on pelvis), mid-thigh, mid-shank, and the forefoot of both lower extremities and were attached with straps. In addition, reflective markers from the MAS were placed on the ASIS (pelvis), sacrum, iliac crest, greater trochanter, thigh, lateral knee, medial knee, shank, lateral ankle, medial ankle, heel, and the first and fifth metatarsal bone $[14,15]$.

\section{Experimental assessment tools and data collection}

\section{D motion analysis system}

In this study, the Qualisys (Qualisys AB, Gothenburg, Sweden, 2012) was used, which consisted with six infrared cameras and software. The system recorded the data from the camera every $100 \mathrm{~Hz}$ and the cameras were calibrated on the test day. This system tracked the position of the retro-reflective markers, calculated the temporal and spatial parameters, and then reconstructed a digital skeleton of the subject by Visual 3D.

The software 'Track Manager' (Track Manager version 2.5, Qualisys, 2012) was used for processing and computing the temporal and spatial parameters of the motion data and the 'Visual 3D Basic' (C-Motion, Rockville, MD, USA, 2012) reconstructed the anatomical structure of the lower body. This program had likewise calculated the angle variations for the subjects' lower extremities, hip, knee and ankle joints [16].

\section{Portable gait analysis system}

The PGAS used in this study consisted of eight 3D inertial measurement unit (IMU) boards as wireless sensors, and one main board as a fusion center. Basically, Euler's angles (roll, pitch, and yaw) can be adopted in the space coordinate to measure the patients' joint angles and walking speeds with the IMU [17], i.e., a roll is a clockwise rotation about the $\mathrm{x}$-axis, a pitch is a clockwise rotation about the y-axis, and a yaw is a clockwise rotation about the z-axis. Each IMU board possessed a magnetometer, an accelerometer, a gyroscope, and a Bluetooth transmitter powered by a rechargeable battery, while the main board included eight Bluetooth receivers and a micro controller unit (MCU) powered by a host computer through a universal asynchronous receiver and transmitter (UART) to a universal serial bus (USB) bridge [18]. Using the sensors attached to the parts of the body, each IMU board could measure the absolute angles of the foot, shank, thigh, and trunk referenced to the axis perpendicular to ground, and transmit the measured angle data to the main board through the air. In this case, the IMU boards were attached to each part of the body for the z-axis to face the direction of movement, so that the precise roll angles could be used to obtain the joint angles. Once the fusion center receives the raw angle data via the Bluetooth receivers, the MCU of the fusion center combined the angle data, and transmited the combined data to the computer connected by a UART and USB bridge. Then, the application software in the computer manipulated the raw data to obtain the relative joint angles of the ankle, knee, and hip, and displayed the raw and manipulated data on the computer screen so that researchers could recognize and use the data for analysis.

\section{Statistical analysis}

The IBM SPSS Statistics for Windows, Version 20.0 (IBM Co., Armonk, NY, USA) used in performance of all statistical analyses. Results were presented as mean \pm standard deviation.

The Shapiro-Wilk test was used to analyze the normal distribution of the medical and general characteristics of subjects. Test-retest reliability between the PGAS and MAS were estimated using the intra-class correlation coefficient (ICCs). Pearson correlation coefficient (r) was used for concurrent validity between systems for the sit to stand joint angles. For all tests, the statistical significance was set at 0.05 .

\section{Results}

\section{Subjects' general characteristics}

For general characteristics of subjects, the sex, age, height, weight, leg length, affected side and stroke type were evaluated (Table 1).

Out of the 16 adults with stroke, there were 9 males $(56.3 \%)$ and 7 females (43.7\%). Considering the stroke type there were 6 with hemorrhagic $(37.5 \%)$ and 10 with in- 
farction $10(62.5 \%)$, in addition, 9 subjects were affected on the left $(56.3 \%)$ and 7 were affected on the right (43.7\%). The mean age was $60.19 \pm 9.01$ years, the mean height was $163.50 \pm 8.80 \mathrm{~cm}$ and the mean weight was $59.44 \pm 11.64 \mathrm{~kg}$. The mean thigh length was $37.50 \pm 2.48 \mathrm{~cm}$ (affected) and

Table 1. General characteristics of subjects

$(\mathrm{N}=16)$

\begin{tabular}{lc}
\hline \multicolumn{1}{c}{ Variable } & Value \\
\hline Male/female & $9(56.3) / 7(43.7)$ \\
Age $(\mathrm{y})$ & $60.19(9.01)$ \\
Height $(\mathrm{cm})$ & $163.50(8.80)$ \\
Weight $(\mathrm{kg})$ & $59.44(11.64)$ \\
Affected side (right /left) & $7(43.7) / 9(56.3)$ \\
Leg length (affected) & \\
$\quad$ Thigh & $37.50(2.48)$ \\
$\quad$ Shank & $44.69(3.16)$ \\
Leg length (unaffected) & $37.31(2.27)$ \\
$\quad$ Thigh & $44.63(3.24)$ \\
$\quad$ Shank & $26.93(1.18)$ \\
MMSE-K (score) & $6(37.5) / 10(62.5)$ \\
Hemorrhage/infarction (n) & $13.00(4.50)$ \\
Onset time (mo) &
\end{tabular}

Values are presented as $\mathrm{n}(\%)$ or mean (SD).

MMSE-K: Mini Mental State Examination-Korea.
$37.31 \pm 2.27 \mathrm{~cm}$ (unaffected). The mean shank length was $44.69 \pm 3.16 \mathrm{~cm}$ (affected) and $44.63 \pm 3.24 \mathrm{~cm}$ (unaffected) respectively.

\section{Test-retest reliability}

As can be seen in Table 2, a reasonable reliability of both systems in variation of joint angle during sit to stand performance is indicated. The ICCs for the wearable sensor-based designed PGAS showed an acceptable test-retest reliability value, with ICC values were ranging from 0.759 to 0.959 . The MAS showed good to excellent test-retest reliability, with ICC values ranging from 0.811 to 0.950 .

\section{Concurrent validity between PGAS and MAS}

The results of concurrent validity between PGAS and MAS for joint angle of sit to stand are as follows (Table 3). A significant positive relationship was observed between PGAS and MAS for variation of joint angle during sit to stand movements $(p<0.01)$. A moderate to high relationship was found in affected hip ( $\mathrm{r}=0.665)$, unaffected hip (0.767), affected knee ( $\mathrm{r}=0.876)$, unaffected knee $(\mathrm{r}=0.886)$, affected ankle $(0.943)$ and unaffected ankle $(0.823)$ respectively.

Table 2. Test-retest reliability

\begin{tabular}{|c|c|c|c|}
\hline Variable & Test & Retest & $\mathrm{ICC}$ \\
\hline \multicolumn{4}{|l|}{ PGAS } \\
\hline Affected hip $\left(^{\circ}\right)$ & 80.687 (9.469) & $77.386(10.579)$ & 0.959 \\
\hline Unaffected hip $\left(^{\circ}\right)$ & 80.421 (9.377) & $81.536(5.786)$ & 0.795 \\
\hline Affected knee $\left(^{\circ}\right)$ & 80.535 (12.729) & $80.627(10.155)$ & 0.897 \\
\hline Unaffected knee $\left(^{\circ}\right)$ & $83.306(9.146)$ & $82.473(5.908)$ & 0.897 \\
\hline Affected ankle $\left(^{\circ}\right)$ & $11.432(5.676)$ & $8.884(6.435)$ & 0.759 \\
\hline Unaffected ankle $\left(^{\circ}\right)$ & $10.742(5.432)$ & $11.428(4.709)$ & 0.914 \\
\hline \multicolumn{4}{|l|}{ MAS } \\
\hline Affected hip $\left(^{\circ}\right)$ & $75.753(9.222)$ & $75.468(10.306)$ & 0.904 \\
\hline Unaffected hip $\left(^{\circ}\right)$ & $76.880(9.382)$ & $76.787(9.520)$ & 0.891 \\
\hline Affected knee $\left(^{\circ}\right)$ & $82.180(10.240)$ & $84.369(8.763)$ & 0.947 \\
\hline Unaffected knee $\left({ }^{\circ}\right)$ & $84.323(9.713)$ & $85.073(7.119)$ & 0.811 \\
\hline Affected ankle $\left(^{\circ}\right)$ & $9.491(6.614)$ & $10.925(6.354)$ & 0.950 \\
\hline Unaffected ankle $\left(^{\circ}\right)$ & $12.068(5.382)$ & $11.793(4.253)$ & 0.833 \\
\hline
\end{tabular}

Values are presented as mean (SD).

ICC: intra-class correlation coefficient, PGAS: portable gait analysis system, MAS: motion analysis system.

Table 3. Concurrent validity between systems for joint angle of sit-to-stand

\begin{tabular}{ccccccc}
\hline Trial & Affected hip $\left({ }^{\circ}\right)$ & Unaffected hip $\left({ }^{\circ}\right)$ & Affected knee $\left({ }^{\circ}\right)$ & Unaffected knee $\left({ }^{\circ}\right)$ & Affected ankle $\left({ }^{\circ}\right)$ & Unaffected ankle $\left({ }^{\circ}\right)$ \\
\hline Test $(\mathrm{r})$ & $0.665^{* *}$ & $0.767^{* *}$ & $0.876^{* *}$ & $0.886^{* *}$ & $0.943^{* *}$ & $0.823^{* *}$ \\
\hline
\end{tabular}

Pearson correlation coefficient $(\mathrm{r})$.

$* * p<0.01$. 


\section{Discussion}

The aim of this study was to evaluate the test-retest reliability of the lower extremity joint angles during STS movement performance with wearable sensors based on the developed PGAS. Test-retest reliability was determined by ICCs. The ICC range of 0.7 to 0.79 was considered 'good' reliability, 0.8 to 0.89 was 'great' reliability, and greater than 0.9 was considered to be 'excellent' reliability [19]. The wearable sensor-based designed PGAS and MAS, which is well-known as gold standard system, demonstrated reasonable test-retest reliability for the assessment of lower extremity joint angles when performing STS (Table 2). Considering the concurrent validity, a significant positive relationship was observed between the wearable sensor-based designed PGAS and the MAS for variation of joint angle during STS movements (Table 3).

Recently, the application of portable systems with wearable sensors in the rehabilitation field has been widely studied due to its convenience [20] and the analysis and application of STS performance in stroke rehabilitation also has been studied with a variety of subjects of different ages. The PGAS based on wearable sensor provides beneficial information as an effective clinical tool in flexible environments. In order to achieve STS, it requires body coordination, balance, stability, and muscle strength [21]. STS is a series of sequence, such as preparatory phase which leads to the seat off state, transfer phase, which is displacement of the center of body mass, the extension phase, and the stabilization phase, which is the final rising [22]. However, individuals post stroke suffer lack of body coordination, muscular activation change, difficulty with postural control and asymmetrical weight bearing [23].

In stroke rehabilitation, analysis of the lower extremities during STS provide information for planning of treatment, clinical progress and outcome measurement, and assist to restore an independent functional levels for stroke survivors. In addition, fall risk estimation is one of the important processes as a prerequisite for ambulation, and the Timed Up and Go test is one of the most useful tools for predicting fall risk. Therefore, the STS movement is a process that should be carried out in advance to perform ambulation, and is a basic element to acquire functional self-reliance for post stroke individuals [24-26]. Furthermore, independence in activity of daily living is associated with quality of life and is important in rehabilitation of the stroke survivor.

This study may eventually lead to a wide range of applica- tion of motion analysis using wearable sensors during STS in in the rehabilitation of poststroke individuals.

\section{Conflict of Interest}

The authors declared no potential conflicts of interest with respect to the authorship and/or publication of this article.

\section{References}

1. Camargos AC, Rodrigues-de-Paula-Goulart F, Teixeira-Salmela LF. The effects of foot position on the performance of the sit-to-stand movement with chronic stroke subjects. Arch Phys Med Rehabil 2009;90:314-9.

2. Lomaglio MJ, Eng JJ. Muscle strength and weight-bearing symmetry relate to sit-to-stand performance in individuals with stroke. Gait Posture 2005;22:126-31.

3. Chou SW, Wong AM, Leong CP, Hong WS, Tang FT, Lin TH. Postural control during sit-to stand and gait in stroke patients. Am J Phys Med Rehabil 2003;82:42-7.

4. Liu M, Chen J, Fan W, Mu J, Zhang J, Wang L, et al. Effects of modified sit-to-stand training on balance control in hemiplegic stroke patients: a randomized controlled trial. Clin Rehabil 2016; 30:627-36.

5. Jung KS, In TS, Cho HY. Effects of sit-to-stand training combined with transcutaneous electrical stimulation on spasticity, muscle strength and balance ability in patients with stroke: a randomized controlled study. Gait Posture 2017;54:183-7.

6. Pollock A, Gray C, Culham E, Durward BR, Langhorne P. Interventions for improving sit-to-stand ability following stroke. Cochrane Database Syst Rev 2014;(5):CD007232.

7. Lee MY, Lee HY. Analysis for sit-to-stand performance according to the angle of knee flexion in individuals with hemiparesis. J Phys Ther Sci 2013;25:1583-5.

8. Tao W, Liu T, Zheng R, Feng H. Gait analysis using wearable sensors. Sensors (Basel) 2012;12:2255-83.

9. Brückner HP, Nowosielski R, Kluge H, Blume H. Mobile and wireless inertial sensor platform for motion capturing in stroke rehabilitation sessions. Paper presented at: 5th IEEE International Workshop on Advances in Sensors and Interfaces IWASI; 2013 Jun 13-14; Bari, Italy.

10. Jonsdottir J, Recalcati M, Rabuffetti M, Casiraghi A, Boccardi S, Ferrarin M. Functional resources to increase gait speed in people with stroke: strategies adopted compared to healthy controls. Gait Posture 2009;29:355-9.

11. Bertolucci PHF, Brucki SMD, Campacci SR, Juliano Y. The mini-mental state examination in an outpatient population: influence of literacy. Arq Neuropsiquiatria 1994;52:1-7.

12. Kwong PW, Ng SS, Chung RC, Ng GY. Foot placement and arm position affect the five times sit-to-stand test time of individuals with chronic stroke. Biomed Res Int 2014. doi: 10.1155/2014/ 636530.

13. Mizuike C, Ohgi S, Morita S. Analysis of stroke patient walking dynamics using a tri-axial accelerometer. Gait Posture 2009;30: 
$60-4$.

14. Bruijn SM, Meijer OG, van Dieën JH, Kingma I, Lamoth CJ. Coordination of leg swing, thorax rotations, and pelvis rotations during gait: the organisation of total body angular momentum. Gait Posture 2008;27:455-62.

15. Cappozzo A, Della Croce U, Leardini A, Chiari L. Human movement analysis using stereophotogrammetry. Part 1: theoretical background. Gait Posture 2005;21:186-96.

16. Cooper G, Sheret I, McMillan L, Siliverdis K, Sha N, Hodgins D, et al. Inertial sensor-based knee flexion/extension angle estimation. J Biomech 2009;42:2678-85.

17. Alandry B, Dumas N, Latorre L, Mailly F, Nouet P. A CMOS multi-sensor system for 3D orientation determination. Paper presented at: 2008 IEEE Computer Society Annual Symposium on VLSI; 2008 Apr 7-9; Montpellier, France.

18. Kok M, Hol JD, Schön TB, Gustafsson F, Luinge H. Calibration of a magnetometer in combination with inertial sensors. Paper presented at: 2012 15th International Conference on Information Fusion; 2012 Jul 9-12; Singapore.

19. Rankin G, Stokes M. Reliability of assessment tools in rehabilitation: an illustration of appropriate statistical analyses. Clin Rehabil 1998;12:187-99.

20. Muro-de-la-Herran A, Garcia-Zapirain B, Mendez-Zorrilla A. Gait analysis methods: an overview of wearable and non-wear- able systems, highlighting clinical applications. Sensors (Basel) 2014;14:3362-94.

21. Galli M, Cimolin V, Crivellini M, Campanini I. Quantitative analysis of sit to stand movement: experimental set-up definition and application to healthy and hemiplegic adults. Gait Posture 2008;28:80-5.

22. Schenkman M, Berger RA, Riley PO, Mann RW, Hodge WA. Whole-body movements during rising to standing from sitting. Phys Ther 1990;70:638-48; discussion 648-51.

23. Duclos C, Nadeau S, Lecours J. Lateral trunk displacement and stability during sit-to-stand transfer in relation to foot placement in patients with hemiparesis. Neurorehabil Neural Repair 2008; 22:715-22.

24. Joshua AM, Karnad SD, Nayak A, Suresh BV, Mithra P, Unnikrishnan B. Effect of foot placements during sit to stand transition on timed up and go test in stroke subjects: a cross sectional study. NeuroRehabilitation 2017;40:355-62.

25. Brunt D, Greenberg B, Wankadia S, Trimble MA, Shechtman O. The effect of foot placement on sit to stand in healthy young subjects and patients with hemiplegia. Arch Phys Med Rehabil 2002;83:924-9.

26. Nyberg L, Gustafson Y. Fall prediction index for patients in stroke rehabilitation. Stroke 1997;28:716-21. 